\title{
Detection of extended-spectrum beta-lactamase-producing E. coli and Klebsiella spp. in effluents of different hospitals sewage in Biratnagar, Nepal
}

\author{
Sanjay Mahato ${ }^{1,2^{*}}$, Ajay Mahato ${ }^{3}$, Elina Pokharel ${ }^{2}$ and Ankita Tamrakar ${ }^{2}$
}

\begin{abstract}
Objective: This study was aimed to determine prevalence and resistance pattern like multidrug resistant (MDR) or ESBL nature of E. coli and Klebsiella spp. from various sewage drain samples with an idea to deliver baseline information that could be utilized for defining guidelines for the treatment of hospital sewages.

Results: Of 10 sewage samples analyzed, 7 (70\%) contained E. coli while $6(60 \%)$ contained Klebsiella. Except one sample, all positive samples contained both E. coli and Klebsiella spp. E. coli isolates were resistant to ampicillin, amoxicillin, cefoxitin, cefuroxime, and cefpodoxime; while $85.7 \%$ were resistant to amoxicillin/clavulanate, ceftazidime, cefotaxime and ceftriaxone. $71.4 \%, 57.1 \%, 42.9 \%$, and $28.6 \%$ were resistant to aztreonam, trimethoprim/sulfamethoxazole, nitrofurantoin, and gentamicin. Most were sensitive to chloramphenicol, ofloxacin, ciprofloxacin, and azithromycin. $85.7 \%$ and $57.1 \%$ of E. coli were MDR and ESBL isolates, respectively. Klebsiella were resistant to ampicillin, amoxicillin, and amoxicillin/clavulanate. $83.4 \%$ of Klebsiella were resistant to cefoxitin. $66.7 \%$ of strains were resistant to cefuroxime, ceftazidime, cefotaxime, ceftriaxone, and cefpodoxime. Klebsiella showed $50 \%$ resistant to aztreonam and trimethoprim/sulfamethoxazole, while 33.3\% were resistant to chloramphenicol, nitrofurantoin, ofloxacin, and ciprofloxacin. Klebsiella were sensitive to azithromycin and gentamicin. $66.7 \%$ and $33.3 \%$ of Klebsiella were MDR and ESBL isolates, respectively.
\end{abstract}

Keywords: Hospital sewage, Klebsiella, E. coli, MDR, ESBL, Water

\section{Introduction}

Sewage designates raw sewage, sewage sludge or septic tank waste containing about $95.5 \%$ water and $0.1 \%$ to $0.5 \%$ organic and inorganic materials. Hospital wastewaters are generated in different sections of hospital like surgery units, ICU, laboratories, patient wards, clinical wards, laundries and possess a quite variable compositions depending on the activities involved [1]. A variety of microorganisms are present in water for examples bacteria, fungi, protozoa etc. Bacteria like Shigella, E. coli, Klebsiella, Vibrio, Salmonella, etc. are found in sewage

\footnotetext{
*Correspondence: Mahato.sanjay@gmail.com

${ }^{1}$ Aasra Research and Education Academy Counsel, Janapriya Tole, Biratnagar-6, Nepal

Full list of author information is available at the end of the article
}

drain water [2]. Coliforms are mainly from family Enterobacteriaceae that are aerobic or facultative aerobic, gram negative, non-spore forming enteric bacilli and basically found in human colon which are introduced into environment by human feces $[3,4]$.

Antibiotics are fractionally metabolized by patients and are then ejected into the hospital sewage. Along with excreta, they pass through sewage system and end up in the environment, mainly in the water area [5]. Hospital sewage discharge a variety of multi resistant bacteria and substances like antimicrobial, pharmaceutical, disinfectants, heavy metals, radioisotopes, and drugs not metabolized by patients [6].

Multiple drug resistance (MDR) for Gram negative and Gram-positive bacteria means "resistant to three or more antimicrobial classes." Extended-spectrum $\beta$-lactamases 
(ESBL) are enzymes that impart resistant to extendedspectrum (third generation) cephalosporins (e.g. ceftazidime, cefotaxime and ceftriaxone) and monobactams (e.g. aztreonam). The most common ESBL producing bacteria are few strains of E. coli and Klebsiella pneumoniae $[7,8]$.

The main aim of this study was to determine prevalence and resistance pattern like MDR or ESBL nature of E. coli and Klebsiella pneumoniae from various sewage drain samples since they can cause serious public health problem. This study could deliver baseline information that could be utilized for defining guidelines for the treatment of hospital sewages.

\section{Main text Methods \\ Sample collection method and characteristics}

During March to October 2018, a total of 10 sewages samples were collected aseptically from different hospitals of Biratnagar city (Table 1). For this, the sample was collected nearby the center of the flow channel, at approximately $10-15 \mathrm{~cm}$ depth from the water surface, where the turbulence was at maximum and the possibility of settling was minimized. Skimming the water surface or dragging the bottle was avoided. The sewage water was first mixed and then $500 \mathrm{ml}$ sample was taken in the sterile high-density polyethylene (HDPE) bottle aseptically. Each sample bottle was properly labelled with date, code number and time with the help of the marker.

\section{Sample transportation and processing}

Samples collected were placed on $4{ }^{\circ} \mathrm{C}$ ice box to inhibit the growth of microorganisms and were immediately transported (within $2 \mathrm{~h}$ ) to microbiology research lab for the analysis. Distilled water was used as control during analysis.
Isolation and identification of E. coli and Klebsiella spp.

The samples collected from the hospital wastewater were serially diluted in $0.85 \%$ saline water and dilution $10^{-2}$ and $10^{-3}$ were inoculated onto Eosin Methylene Blue (EMB) Agar and MacConkey Agar for Escherichia coli and Klebsiella spp. by spread plate method and were incubated aerobically at $37^{\circ} \mathrm{C}$ for $24-48 \mathrm{~h}$. After incubation, colonies were picked on their colony morphology like colonial appearance, size, elevation, color, margin, and opacity. All the selected colonies were, then, subcultured on nutrient agar plate to obtain pure culture for the microscopic and biochemical identification. TSI (triple sugar iron), SIM (sulfate/indole/motility), Methyl Red test, Voges-Proskauer test, citrate agar, catalase test, oxidase test, and urea hydrolysis test were performed to identify the organisms [9].

\section{Antimicrobial susceptibility tests}

The identified isolates of Escherichia coli and Klebsiella spp. were submitted to antimicrobial susceptibility testing according to the guidelines of the Clinical and Laboratory Standards Institute [10]. The isolates were inoculated onto Mueller-Hinton agar medium using turbidity of 0.5 McFarland standard. The following antimicrobial disk (Himedia, Mumbai, India) were used: Ampicillin (AMP) $(10 \mu \mathrm{g})$, Amoxicillin (AMX) $(10 \mu \mathrm{g})$, Amoxicillin/ clavulanate (AMC) $(20 / 10 \mu \mathrm{g})$, Cefoxitin (CX) $(30 \mu \mathrm{g})$, Ceftazidime (CAZ) $(30 \mu \mathrm{g})$, Ceftriaxone (CTR) $(30 \mu \mathrm{g})$, Cefpodoxime (CPD) $(10 \mu \mathrm{g})$, Cefuroxime (CXM) $(30 \mu \mathrm{g})$, Aztreonam (AT) $(30 \mu \mathrm{g})$, Chloramphenicol (C) $(30 \mu \mathrm{g})$, Azithromycin (AZM) $(15 \mu \mathrm{g})$, Gentamicin (GEN) $(30 \mu \mathrm{g})$, Ciprofloxacin (CIP) $(5 \mu \mathrm{g})$, Ofloxacin (OF) $(5 \mu \mathrm{g})$, Nitrofurantoin (NIT) $(300 \mu \mathrm{g})$, and Trimethoprim/sulfamethoxazole (COT) $(1.25 / 23.75 \mu \mathrm{g})$. The swabbed MHA plates with the discs were incubated at $37^{\circ} \mathrm{C}$ for $24 \mathrm{~h}$. Zone of inhibition was measured and interpreted using the standard chart [10]. Due to unavailability of ATCC culture,

Table 1 Sample collection detail of hospitals from Biratnagar, Nepal

\begin{tabular}{|c|c|c|c|c|c|c|}
\hline Sample & Date & Location & Code & Klebsiella & E. coli & Code \\
\hline 1 & 0125 & Saptakoshi Hospital & S1LP & + & + & S1P \\
\hline 2 & 0125 & Saptakoshi Hospital & S2LP & + & + & $\mathrm{S} 2 \mathrm{G}$ \\
\hline 3 & 0131 & Tulasa Mother and Child Hospital & S3LP & + & + & S3G \\
\hline 4 & 0131 & Max International Hospital & S4 & - & - & S4 \\
\hline 5 & 0204 & Lifeguard Hospital & S5LP & + & + & S5G \\
\hline 6 & 0406 & Morang Sahakari Hospital & S6 & - & - & S6 \\
\hline 7 & 0406 & Morang Sahakari Hospital & S7 & - & - & S7 \\
\hline 8 & 0408 & Koshi Zonal Hospital & S8LP & + & + & S8G \\
\hline 9 & 0408 & Koshi Zonal Hospital & S9 & - & + & S9LP \\
\hline 10 & 0408 & Koshi Zonal Hospital & S10LP & + & + & S10LP \\
\hline
\end{tabular}


sensitive E. coli and Klebsiella pneumoniae strains with established antibiogram were used as control.

\section{Criterion for multidrug resistance}

Isolates which demonstrated the resistance to at least one agent in three or more classes of the drug were defined as multidrug resistant (MDR) [10, 11].

\section{ESBL detection}

Isolates exhibiting a zone of inhibition of growth for ceftazidime and ceftriaxone $\leq 22 \mathrm{~mm}$ and $\leq 25 \mathrm{~mm}$, respectively, were submitted to the combined disc test to check for ESBL-producing strains [12]. The combined disc methodology used to detect ESBL-producing $E$. coli and Klebsiella spp. was performed as per CLSI [10]. The antimicrobials used were ceftazidime $(30 \mu \mathrm{g})$ and ceftazidime/clavulanic acid $(30 / 10 \mu \mathrm{g})$, and cefotaxime $(30 \mu \mathrm{g})$ and cefotaxime/clavulanic acid $(30 / 10 \mu \mathrm{g})$. Results were interpreted as per the criteria established by the CLSI [10]. An increase of $5 \mathrm{~mm}$ in a zone of inhibition of growth for combined drugs to ceftazidime or cefotaxime were confirmatory for ESBL-producing strains [10, 12].

\section{Multiple antibiotic resistance (MAR) index}

MAR index is the number of antibiotics to which test isolate displayed resistance divided by the total number of antibiotics to which the test organism has been evaluated for sensitivity. MAR index for each isolate was calculated as per the guidelines of Krumperman [13].

\section{Data analysis}

The data were statistically analyzed using Statistical Package for Social Sciences (SPSS v21) software package. Chi square test at $\mathrm{p}$-value $<0.05$ was considered statistically significant.

\section{Results}

Out of 10 samples analyzed, 7 (70\%) contained E. coli while 6 (60\%) contained Klebsiella. Except one sample, all positive samples contained both E. coli and Klebsiella spp.

Colonies with green metallic sheen on EMB agar on further analysis were confirmed to be $E$. coli. Microscopic examinations revealed them to be gram negative non-capsulated bacilli (1SH, 2SH, 3TMC, 5LG, 8KZ, $9 \mathrm{KZ}, 10 \mathrm{KZ}$ ). All the 7 isolates were motile, non-hydrogen sulfide producers; VP, citrate, oxidase negative while was indole, methyl red, catalase, urease, TSI (acid/acid with gas) positive. Pink colored, highly mucoid colonies in EMB Agar on further examinations were found to be Klebsiella pneumoniae. Microscopic examinations revealed them to be gram negative capsulated bacilli (1SH, 2SH, 3TMC, 5LG, $8 \mathrm{KZ}, 10 \mathrm{KZ})$. All the 6 isolates were non-motile, non-hydrogen sulfide producers; indole, MR, oxidase negative while was VP, citrate, catalase, urease, TSI (acid/acid with gas) positive.

Out of 7 samples $(\mathrm{n}=7)$ of $E$. coli, all the isolates were resistant to ampicillin, amoxicillin, cefoxitin, cefuroxime, and cefpodoxime. $85.7 \%$ of E. coli were resistant to amoxicillin/clavulanate and cephalosporins like ceftazidime, cefotaxime and ceftriaxone. The resistance shown by $E$. coli to aztreonam, Trimethoprim/sulfamethoxazole, nitrofurantoin, and gentamicin were $71.4 \%, 57.1 \%, 42.9 \%$, and $28.6 \%$, respectively (Table 2 ). $14.3 \%$ of strains were resistant to chloramphenicol and fluoroquinolones like ofloxacin, ciprofloxacin. All the strains were sensitive to azithromycin. Out of 7 isolates, 6 (85.7\%) of E. coli $(1 \mathrm{SH}$, 2SH, 3TMC, 5LG, 9KZ, 10KZ) were multidrug resistant (MDR) bacteria. Notably 4 isolates (57.1\%) of E. coli (1SH, $2 \mathrm{SH}, 9 \mathrm{KZ}, 10 \mathrm{KZ}$ ) were confirmed as ESBL producing isolates.

Klebsiella pneumoniae were resistant to ampicillin, amoxicillin, and amoxicillin/clavulanate. $83.4 \%$ of Klebsiella were resistant to cefoxitin; while $66.7 \%$ were resistant to cefuroxime, ceftazidime, cefotaxime, ceftriaxone, and cefpodoxime (Table 2). Klebsiella showed 50\% resistant to aztreonam and Trimethoprim/sulfamethoxazole. $33.3 \%$ of strains were resistant to chloramphenicol, nitrofurantoin, ofloxacin, and ciprofloxacin. Only 16.7\% of strains were resistant to azithromycin while were fully sensitive to gentamicin. Out of 6 Klebsiella, only 4

Table 2 Antibiotic susceptibility pattern of Klebsiella and $E$. coli in percentage

\begin{tabular}{lll}
\hline Antibiotics & \multicolumn{2}{l}{ Resistance percentage (\%) } \\
\cline { 2 - 3 } & Klebsiella & E. coli \\
\hline Ceftazidime & 66.7 & 85.7 \\
Cefotaxime & 66.7 & 85.7 \\
Cefoxitin & 66.7 & 57.1 \\
Ceftriaxone & 66.7 & 85.7 \\
Cefpodoxime & 66.7 & 100 \\
Cefuroxime & 66.7 & 85.7 \\
Chloramphenicol & 50 & 14.3 \\
Gentamicin & 0 & 28.6 \\
Amoxicillin/clavulanate & 100 & 14.3 \\
Ampicillin & 100 & 100 \\
Amoxicillin & 100 & 100 \\
Aztreonam & 50 & 71.4 \\
Ciprofloxacin & 33.3 & 14.3 \\
Ofloxacin & 33.3 & 14.3 \\
Azithromycin & 16.7 & 0 \\
Nitrofurantoin & 66.7 & 0 \\
Trimethoprim/sulfamethoxazole & 50 & 57.1 \\
\hline
\end{tabular}


(66.7\%) (1SH, 2SH, 3TMC, 8KZ) were MDR. Two isolates $(33.3 \%)$ of Klebsiella $(2 \mathrm{SH}, 8 \mathrm{KZ})$ were confirmed to be ESBL producing isolates.

Multiple antibiotic resistance (MAR) indices of bacteria revealed that none of E. coli and Klebsiella were susceptible or resistant to all the seventeen tested drugs (Fig. 1). Of all 7 E. coli, 1 (14.3\%) was resistant to 6 drugs $(\mathrm{MARI}=0.353), 1(14.3 \%)$ was resistant to 9 drugs (MARI $=0.529), 3(42.9 \%)$ were resistant to 11 drugs $($ MARI $=0.647)$, and $2(28.6 \%)$ was to 14 drugs $($ MARI $=0.824)$. Of all 6 Klebsiella, $1(16.7 \%)$ was resistant to 3 drugs (MARI $=0.176), 1(16.7 \%)$ was resistant to 4 drugs (MARI $=0.235), 2(33.3 \%)$ were resistant to 10 drugs (MARI $=0.588), 1(16.7 \%)$ was resistant to 15 drugs $(\mathrm{MARI}=0.882)$, and $1(16.7 \%)$ was to 16 drugs $($ MARI $=0.941)$.

There is no significant relationship between the type of bacterial strains (like E. coli and Klebsiella) and their response to the antibiotics at $\mathrm{df}=1, \mathrm{p}=0.05$.

\section{Discussion}

The main aim of this study was to determine prevalence and resistance pattern like MDR or ESBL nature of $E$. coli and Klebsiella pneumoniae from various sewage drain samples. Presence of 70\% of E. coli and 60\% of Klebsiella in sewage may have direct link with the human feces in many cases [14]. Sewage mass is liquid mass containing excessive amount of organic matter which acts as a nutrient medium for all the bacteria [15]. Excessive number of MDR and ESBL E. coli and Klebsiella show that drainage system of Biratnagar hospitals is highly infectious and life threatening if contaminated with water and food [16].

The result of E. coli showing $100 \%$ resistance to ampicillin, amoxicillin, cefoxitin, cefuroxime, and cefpodoxime was higher than Belachew et al. [17] showing 91.3\% resistance to ampicillin, $70 \%$ resistance to cefuroxime and ceftriaxone, Cefpodoxime (74\%), amoxicillin/clavulanate (52\%), cefoxitin (43\%), ceftazidime (65\%). In this study, $85.7 \%$ of E. coli were resistant to amoxicillin/ clavulanate, ceftazidime, cefotaxime and ceftriaxone. Resistance to nitrofurantoin was similar in Belachew et al. [17]. Resistance to aztreonam and chloramphenicol were higher than the findings of Florica et al. [18]. On the contrary, resistance to trimethoprim/sulfamethoxazole (57.1\%), gentamicin (28.6\%) and ciprofloxacin (14.3\%) in our study was lower than Belachew et al. [17] showing $67 \%, 43 \%$, and $52 \%$, respectively. It has been observed that none of the hospitals in Biratnagar have waste treatment system as a result, $85.7 \%$ of E. coli species had multi-drug resistance, which is, higher compared to previously reported results in Ethiopia (78\%) [17] and Romania $(60.34 \%)[18]$. Such a high resistance rate may be a

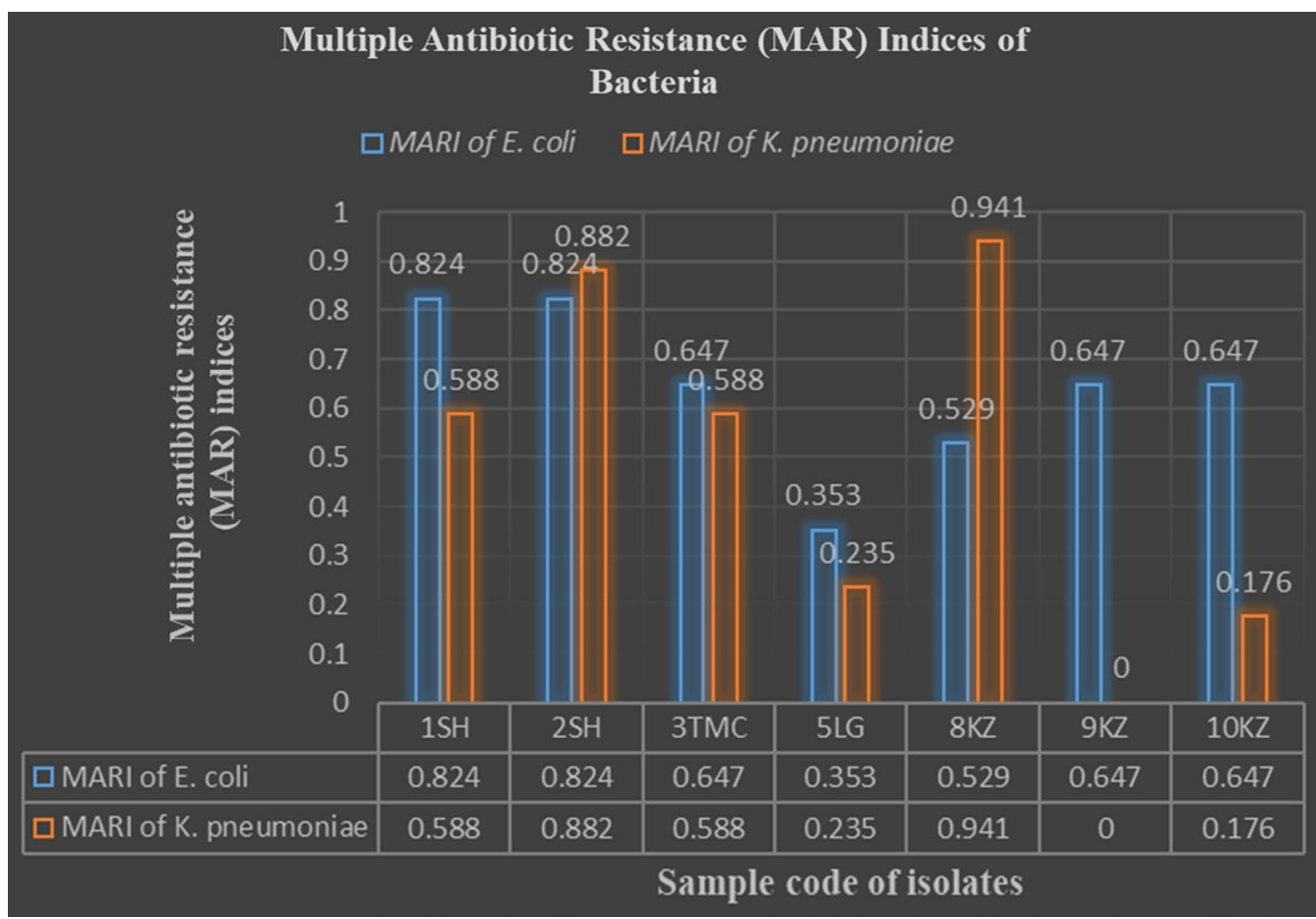

Fig. 1 Multiple antibiotic resistance (MAR) indices of bacteria. MAR indices of bacteria revealed that none of E. coli and Klebsiella were susceptible or resistant to all the seventeen tested drugs 
result of poor waste management practice, lack of treatment plants for healthcare institutions and poor antimicrobial usage in Biratnagar.

Klebsiella pneumoniae showed $100 \%$ resistance to ampicillin and amoxicillin/clavulanate which was higher than the study of Ethiopia (94\%) [17] and Romania (70.7\%) [18]. Resistance to penicillin antibiotics like ampicillin has become very common in the world and our finding is in line with this evidence. Resistance of $66.7 \%$ for ceftazidime, cefotaxime, and ceftriaxone was higher than the findings of Romania [18] as 8.6\%, 17.2\% and $13.8 \%$, respectively. Resistance shown by Trimethoprim/sulfamethoxazole (50\%), chloramphenicol (33.3\%) and ciprofloxacin $(33.3 \%)$ were much higher than found in Romania as $22.4 \%, 5.2 \%$ and $6.9 \%$. Similarly, the resistance to cefoxitin, cefuroxime, cefpodoxime, and nitrofurantoin in this study was found to be much higher than other studies [17]. A high rate of MDR (66.7\%) was observed for Klebsiella spp. which was higher than results reported in Ethiopia (40.5\%) [17], Romania (33\%) [18] and Mexico (50\%) [19]. However, MDR rate of the current finding was lower than previously reported results in Brazil (77.5\%) [20]. Such variation may be due to the difference in antimicrobial use and availability of waste treatment system in hospital sewage [17].

\section{Conclusion}

This study builds the importance to enquire the involvement of hospital liquid waste discharge in the development and distribution of antibiotics resistance in the environment. There is rise in resistant bacteria like $E$. coli and Klebsiella in hospital wastewater. The government must implement some rules and laws for proper treatment of hospital wastewater before entry to main municipal wastewater. Sewage treatment plant must be established in hospital for their effluents and sludge coming from the hospital's units.

\section{Limitations}

The standard strain E. coli (ATCC 25922) and K. pneumoniae (ATCC 13883) could not be used.

\footnotetext{
Abbreviations

MDR: multiple drug resistance; ESBL: extended-spectrum $\beta$-lactamases; ICU: intensive care unit; HDPE: high density polyethylene; EMB: Eosin Methylene Blue; TSI: triple sugar iron; SIM: sulfate/indole/motility; CLSI: Clinical and Laboratory Standards Institute; MHA: Mueller-Hinton agar; AMP: ampicillin; AMX: amoxicillin; AMC: amoxicillin/clavulanate; CX: cefoxitin; CAZ: ceftazidime; CTR: ceftriaxone; CPD: cefpodoxime; CXM: cefuroxime; AT: aztreonam; C: chloramphenicol; AZM: azithromycin; GEN: gentamicin; CIP: ciprofloxacin; OF: ofloxacin; NIT: nitrofurantoin; COT: trimethoprim/sulfamethoxazole; $\mu \mathrm{g}$ : microgram; ml: milliliter; MAR: multiple antibiotic resistance; MAR: multiple antibiotic resistance indices; WHO: World Health Organization.
}

\section{Acknowledgements}

We sincerely appreciate the visionary efforts and inputs of AASRA Research and Education Academy Counsel for making this study possible. We are also thankful to those individuals who made this study possible through their contribution by any means.

\section{Authors' contributions}

SM and AM were involved in planning the research, study design, analysis, and writing the manuscript; SM, EP and AT were involved in laboratory work and data collection and compiling, preparation of report; SM and AM were involved in analysis, review and editing. All authors read and approved the final manuscript.

\section{Funding}

No fund/grant was received for the research work.

\section{Availability of data and materials}

All the required data and material of research is given in the manuscript.

\section{Ethics approval and consent to participate}

Research approval was taken from AASRA Research and Education Academy Counsel, Biratnagar and Microbiology Department of MMAMC, Biratnagar, Nepal.

\section{Consent for publication}

Not applicable.

\section{Competing interests}

The authors declare that they have no competing interests.

\section{Author details}

${ }^{1}$ Aasra Research and Education Academy Counsel, Janapriya Tole, Biratnagar-6, Nepal. ${ }^{2}$ Department of Microbiology, Mahendra Morang Adarsha Multiple Campus, Tribhuvan University, Biratnagar, Nepal. ${ }^{3}$ Department of Orthopaedics, Birat Medical College and Teaching Hospital, Biratnagar, Nepal.

Received: 12 August 2019 Accepted: 28 September 2019

Published online: 04 October 2019

\section{References}

1. Aurelien Bde H, Sylvie B, Alain D, Jerome G, Yves P. Ecotoxicological risk assessment linked to the discharge by hospitals of bio-accumulative pharmaceuticals into aquatic media: the case of mitotane. Chemosphere. 2013;93(10):2365-72.

2. Al-Gheethi AA, Efaq AN, Bala JD, NorliM I, Abdel-Monem MO, Ab Kadir MO. Appl Water Sci. 2018:8:74.

3. Manyi-Loh CE, Mamphweli SN, Meyer EL, Makaka G, Simon M, Okoh Al. An overview of the control of bacterial pathogens in cattle manure. Int J Environ Res Public Health. 2016;13(9):843.

4. Martin NH, Trmčić A, Hsieh TH, Boor KJ, Wiedmann M. The evolving role of coliforms as indicators of unhygienic processing conditions in dairy foods. Front Microbiol. 2016;7:1549.

5. Mohameda HSA, Uswege M, Robinson HM. Correlation between antibiotic concentrations genes contaminations at Mafisa wastewater treatment plant in Morogoro Municipality, Tanzania. Glob Environ Health Saf. 2018;2(1):5

6. Fekadu S, Merid Y, Beyene H, Teshome W, Gebre-Selassie S. Assessment of antibiotic- and disinfectant-resistant bacteria in hospital wastewater, south Ethiopia: a cross-sectional study. J Infect Dev Ctries. 2015;9:149-56.

7. Rawat D, Nair D. Extended-spectrum $\beta$-lactamases in Gram Negative Bacteria. J Glob Infect Dis. 2010;2(3):263-74.

8. Mahato S, Mahato A, Yadav J. Prevalence and identification of uropathogens in eastern Nepal and understanding their antibiogram due to multidrug resistance and Esbl. Asian Pac J Microbiol Res. 2018;2(1):09-17.

9. Cheesbrough M. Biochemical tests to identify bacteria. District laboratory practice in tropical countries, Part II. 2nd ed. New York: Cambridge University Press; 2009. p. 45-58. 
10. Clinical and Laboratory Standards Institute. Performance standards for antimicrobial susceptibility testing: 26th informational supplement. Wayne: CLSI; 2016.

11. Magiorakos AP, Srinivasa A, Carey RB, Carmeli Y, Falagas ME, Giske CG. Multidrug-resistant, extensively drug-resistant and pandrug-resistant bacteria: an international expert proposal for interim standard definitions for acquired resistance. Clin Microbiol Infect Dis. 2011;18:268-81.

12. Kumar D, Singh AK, Ali MR, Chander Y. Antimicrobial susceptibility profile of extended spectrum $\beta$-Lactamase (ESBL) producing escherichia coli from various clinical samples. Infect Dis. 2014;7:1-8.

13. Krumpermann $\mathrm{PH}$. Multiple antibiotics resistance indexing of E. coli to Identify high risks sources of faecal contamination of foods. Appl Environ Microbiol. 1983;46(1):165-70.

14. World Health Organization. Guidelines for drinking water quality. 4th ed. Geneva: World Health Organization; 2017.

15. Abdel-Raouf N, Al-Homaidan AA, Ibraheem IB. Microalgae and wastewater treatment. Saudi J Biol Sci. 2012;19(3):257-75.

16. Korzeniewska E, Korzeniewska A, Harnisz M. Antibiotic resistant Escherichia coli in hospital and municipal sewage and their emission to the environment. Ecotoxicol Environ Saf. 2013. https://doi.org/10.1016/j. ecoenv.2013.01.014
17. Belachew T, Mihret A, Legesse T, Million Y, Desta K. High level of drug resistance by gram-negative bacteria from selected sewage polluted urban rivers in Addis Ababa, Ethiopia. BMC Res Notes. 2018;11(1):524. https://doi.org/10.1186/s13104-018-3622-0.

18. Florica M, Luminita M, loana S, Veronica L. Antibiotic resistance markers among gram-negative isolates from wastewater and receiving rivers in South Romania. Roman Biotechnol Lett. 2015;20(1):10055-69.

19. Delgado GMCE, Tamez GP, Gomez FR, Zavala DSFJ, Gilberto EV, Guadalupe Virginia NM, et al. Multidrug-resistant bacteria isolated from surface water in Bassaseachic Falls National Park, Mexico. Int J Environ Res Public Health. 2016;13:597. https://doi.org/10.3390/ijerph13060597.

20. de Sousa JA, Silva-Souza ÂT. Bacterial community associated with fish and water from Congonhas River, Sertaneja, Paraná, Brazil. Biol Technol Int J. 2001;44(4):373-81.

\section{Publisher's Note}

Springer Nature remains neutral with regard to jurisdictional claims in published maps and institutional affiliations.
Ready to submit your research? Choose BMC and benefit from:

- fast, convenient online submission

- thorough peer review by experienced researchers in your field

- rapid publication on acceptance

- support for research data, including large and complex data types

- gold Open Access which fosters wider collaboration and increased citations

- maximum visibility for your research: over $100 \mathrm{M}$ website views per year

At BMC, research is always in progress.

Learn more biomedcentral.com/submissions 